\title{
An unusual presentation of pneumothorax associated with cystic lung metastasis from epithelioid sarcoma: A case report and review of the literature
}

\author{
MANABU HOSHI, NAOTO OEBISU, TADASHI IWAI, \\ MAKOTO IEGUCHI, YOSHITAKA BAN and HIROAKI NAKAMURA
}

Department of Orthopaedic Surgery, Osaka City University Graduate School of Medicine, Abeno, Osaka 545-8585, Japan

Received October 23, 2017; Accepted January 17, 2018

DOI: $10.3892 / 01.2018 .7868$

\begin{abstract}
Circumscribed solid nodules are common in pulmonary metastases from sarcoma, but cystic pulmonary metastases from sarcomas are extremely rare. An unusual case of a 33-year-old man presenting with left spontaneous pneumothorax in association with cystic pulmonary metastases from an epithelioid sarcoma in the left forearm was discussed in the present study. The patient underwent wide resection of an epithelioid sarcoma in the left forearm 6 years prior. Periodical computed tomography revealed a gradual increase in the number and size of bilateral multiple cystic pulmonary metastases despite repeated chemotherapy treatment. At 33 years of age, the patient visited the outpatient department for sudden left chest pain and dyspnea. Plain radiography demonstrated spontaneous pneumothorax in the left lung; chest tube insertion and pleurodesis were successfully performed thereafter. At the last follow-up, 24 months after spontaneous pneumothorax, he is still alive and receiving chemotherapy treatment. Spontaneous pneumothorax is a common complication associated with cystic pulmonary metastases from epithelioid sarcoma. Orthopedic oncologists need to be aware of this unusual sarcomatous metastasis during the follow-up of advanced epithelioid sarcoma.
\end{abstract}

\section{Introduction}

Pulmonary metastases from sarcomas form well-circumscribed nodules. The incidence of cystic pulmonary metastasis caused by a soft tissue tumor is extremely rare. When a pulmonary cyst is not accompanied by nodules, it may not be readily identified as a pulmonary metastasis (1). Previously, seven cases

Correspondence to: Dr Manabu Hoshi, Department of Orthopaedic Surgery, Osaka City University Graduate School of Medicine, 1-4-3 Asahi-Machi, Abeno, Osaka 545-8585, Japan E-mail: hoshi@med.osaka-cu.ac.jp

Key words: epithelioid sarcoma, cyst, lung metastasis, pneumothorax with pulmonary cystic metastases from an epithelioid sarcoma have been reported in the English literature (1-7). We report the case of an epithelioid sarcoma of the left forearm, with cystic metastasis to the lung in a 33-year-old man presenting with left spontaneous pneumothorax during repeated chemotherapy treatment; we also reviewed the literature. The clinical behavior of this rare presentation of epithelioid sarcoma is discussed.

This patient and his families were informed that the data from his case would be submitted for publication, and provided consent.

\section{Case report}

A 33-year old man presented with sudden dyspnea and chest pain on the left side. The patient had undergone wide resection of an epithelioid sarcoma in the left forearm (Fig. 1) and pre- and post-operative chemotherapy treatment 6 years prior. Four years ago, he complained of continuous bloody sputum. A solid nodule of sarcomatous metastasis appeared in the right lung, and histological examination via bronchoscopy confirmed the pulmonary metastases from the epithelioid sarcoma (Fig. 2). Following histological confirmation, he started receiving repeated chemotherapy treatments, composed of doxorubicin (DOX; $60 \mathrm{mg} / \mathrm{m}^{2}$ )/ifosfamide (IFM; $10 \mathrm{~g} / \mathrm{m}^{2}$ ), ifosfamide (IFM; $15 \mathrm{~g} / \mathrm{m}^{2}$ ), docetaxel (DTX; $\left.75 \mathrm{mg} / \mathrm{m}^{2}\right) /$ gemcitabine (GEM; $900 \mathrm{mg} / \mathrm{m}^{2}$ ), and methotrexate (MTX; $10 \mathrm{~g} / \mathrm{m}^{2}$ ). Despite repeated chemotherapy treatments, multiple small, thin-walled cystic metastases in bilateral lungs appeared on computed tomography (CT) examination (Fig. 3), and the number and size of cystic metastases increased in 4 years.

Plain radiography and $\mathrm{CT}$ displayed spontaneous pneumothorax in the left lung (Fig. 4). Chest tube placement and pleurodesis in the right lung were performed. Complete pulmonary expansion was achieved. Thereafter, he began receiving chemotherapy again, and 2 years have passed since the recovery from pneumothorax. At the last follow-up, the patient was living with multiple cystic pulmonary metastases, and he developed bone metastases in the rib, sacrum, and left femur; cystic pulmonary metastases gradually appeared, and radiotherapy was administered to each bone metastasis. 


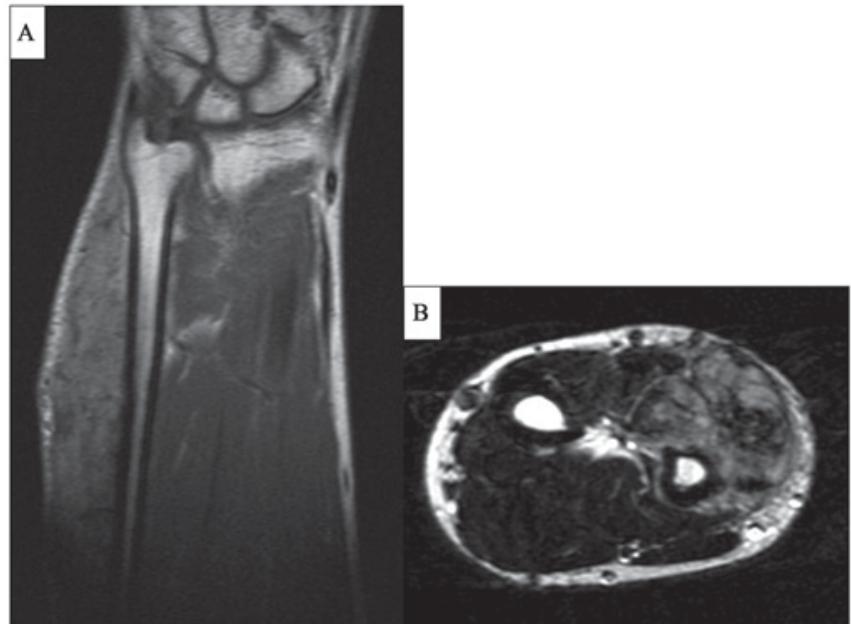

Figure 1. The primary tumor of the patient in the left forearm on coronal view of (A) the enhanced T1-weighted image; the tumor sounded the left ulna and extended to the subcutaneous on axial view of (B) the T2-weighted image.

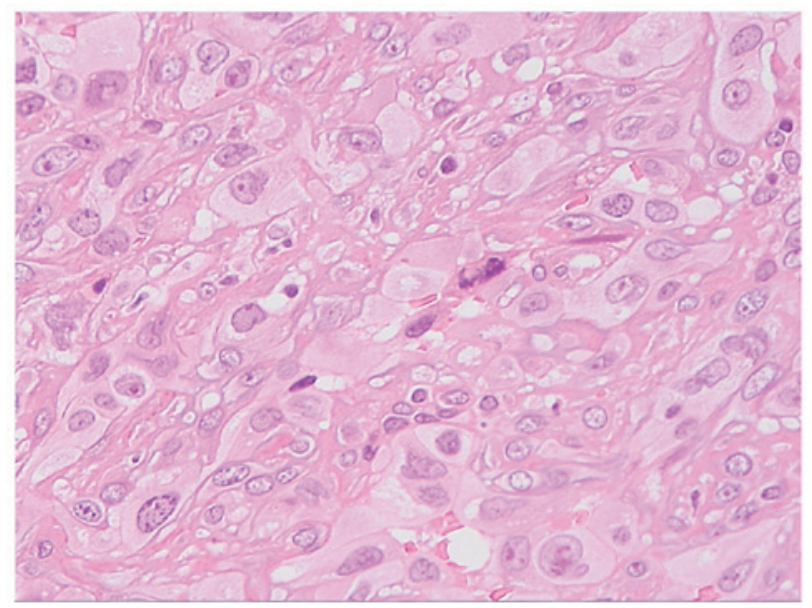

Figure 2. Microscopically, the tumor was composed of nests and sheets of large epithelioid cells, which is consistent with epithelioid sarcoma (hematoxylin and eosin staining; magnification, $\mathrm{x} 400$ ).

\section{Discussion}

Epithelioid sarcoma is a rare malignant tumor with an incidence of 0.6-1.0\% among sarcomas (8,9), and has an extremely poor prognosis. Metastases develop in $40-50 \%$ of patients, usually after repeated recurrences, and frequently involve the lung and regional lymph node (10). Pulmonary metastases from sarcomas usually appear on CT as small, solid, rounded peripheral nodules. Pulmonary cystic metastasis from sarcoma is extremely rare. Only seven previous studies in English on cystic pulmonary metastases from epithelioid sarcoma have been described (1-7).

In our patient, the cystic lesion was not evident on chest radiograpy, but was well visible with $\mathrm{CT}$. CT has been shown to be more sensitive in the detection of small metastatic foci. Small cystic pulmonary lesions are also best visualized with CT. Cystic change associated with primary lung cancer is relatively frequent. Cystic pulmonary metastases usually form a thin smooth wall similar to an emphysematous bulla or a pneumatocele (2). The accurate differential diagnosis of a
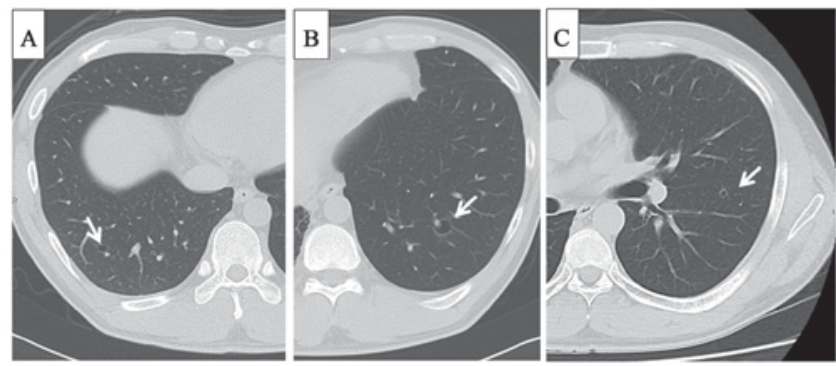

Figure 3. Pulmonary computed tomography demonstrated cystic pulmonary metastases (white arrows) in the right lung (A) and left lung (B and C).

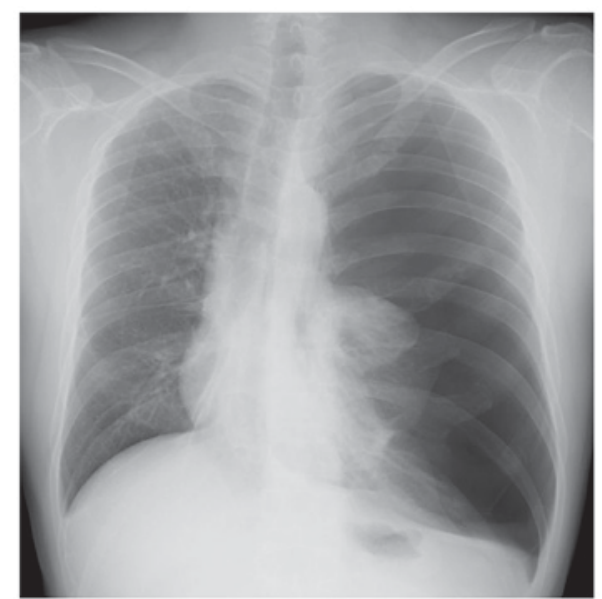

Figure 4. Plain radiograpy showing pneumothorax in the left lung.

cavity metastasis from a benign bulla and primary lung cancer is critically important. Metastatic tumor nodules are easily recognizable when many solid tumor nodules are also present. When few or no solid lesions are seen, however, cavity tumor nodules must be differentiated from primary lung cancer, primary benign cystic pulmonary disease $(2,11,12)$. Ultimately, the detailed pathological diagnosis is mandatory to determine a cystic change in sarcoma.

An additional complication of pulmonary metastases is the occurrence of spontaneous pneumothorax. Spontaneous pneumothorax associated with metastatic tumor is a rare condition, despite that sarcomatous pulmonary metastases are relatively common. According to the previous paper by Hoag et al (13), the incidence of pneumothorax was between 5 to 10 per 100,000 per year, and the prevalence of spontaneous pneumothorax in sarcoma was calculated to be $1.9 \%$. Additionally, Hoag et al (13) reviewed 126 cases with pneumothorax secondary to sarcomatous metastases, and the common histologies were found to be osteogenic sarcoma (31.4\%), angiosarcoma (18.3\%), synovial sarcoma (8.5\%), and epithelioid sarcoma (1.3\%). Transpleural rupture of subpleural cysts may result in the development of pneumothoraces $(14,15)$.

On reviewing the previously reported seven cases of cystic pulmonary metastases from epithelioid sarcoma (Table I), most cases were found to have multiple metastases in bilateral lungs, and pneumothorax was reported to be a frequent complication (85.7\%). Four cases with cystic pulmonary metastases presented with bilateral pneumothorax, two cases presented 


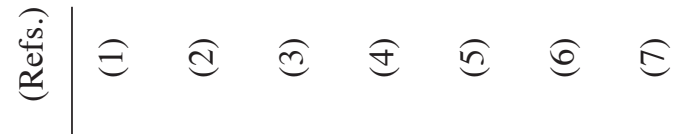

छี

ठั.

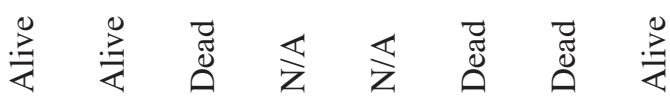

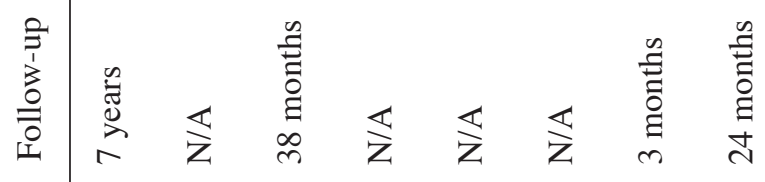

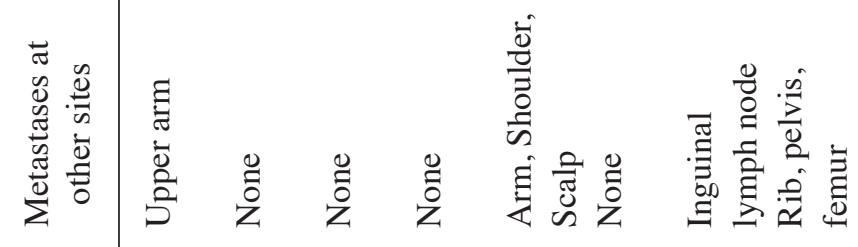

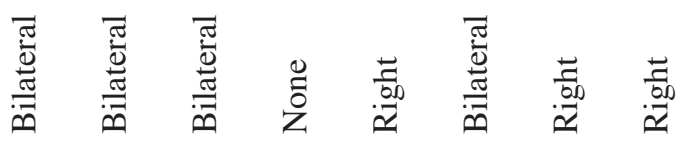

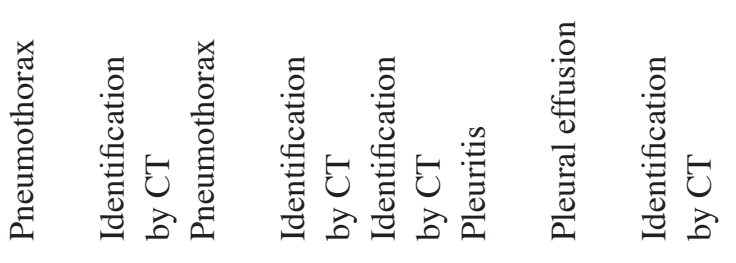

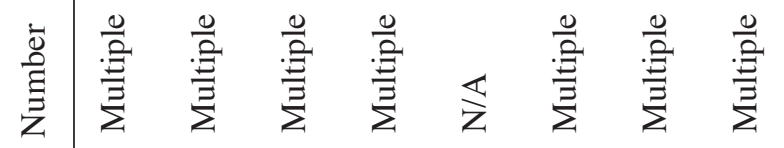

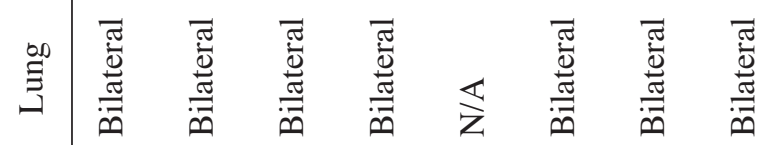

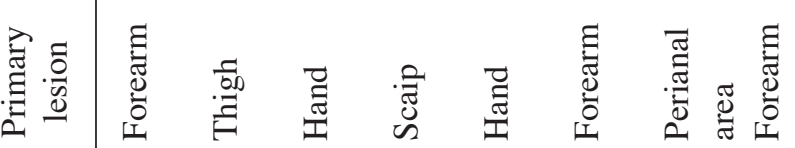

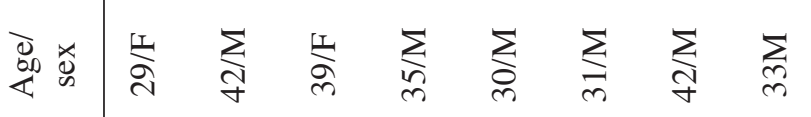

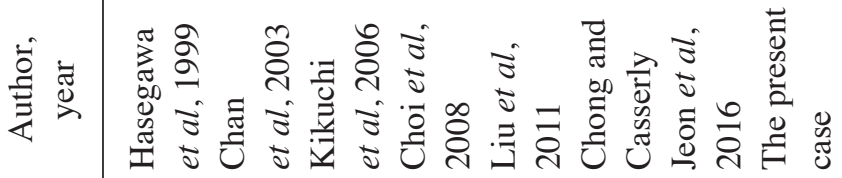


with unilateral pneumothorax, and only one presented without pneumothorax.

Regarding the treatment of pneumothorax, Chan et al (2) reported a case of recurrent spontaneous pneumothorax requiring surgery for the cystic lesions. Traweek et al (14) proposed that these lesions must be surgically removed from the patients. On reviewing 153 cases of spontaneous pneumothorax-complicated sarcoma by Hoag et al (13), chest tube placement was performed most commonly (73.2\%), followed by thoracic surgery $(36.1 \%)$, pleurodesis $(21.6 \%)$, and aspiration (13.4\%).

In the present case, pleurodesis was successfully achieved. In the previous seven cases with pneumothorax of epithelioid sarcoma (Table I), thoracotomies were required in four cases, and pleurodesis in two cases. Considering the treatment of pneumothorax associated with cystic metastases from epithelioid sarcoma, chest tube placement must be performed immediately, and then thoracic surgery should be performed if all lung metastases are locally resectable. In patients with multiple lung metastases, it may be advisable to start systemic chemotherapy promptly at first in pleurodesis. If pleurodesis is not effective, thoracic surgery is considerable.

Although, lung metastasis occasionally undergoes a cystic change from a solid nodule due to central necrosis in the natural cancer course, in the present case, the patient continued to receive repeated chemotherapies of DOX/IFM, IFM, DTX/GEM, and MTX. It is suggested that chemotherapeutic agents are likely to be effective for microscopic lung metastases and responsible for the discharge of necrotic materials inside, resulting in cystic changes in the lung. Moreover, the necrosis of a peripherally located pulmonary metastasis in response to chemotherapy is likely to be responsible for pneumothorax (14).

After spontaneous pneumothorax, Hoag et al (13) reported that the prognoses of sarcomas after pneumothorax is generally poor, with $50 \%$ mortality between 4 and 5 months, and $75 \%$ with 1-year mortality; furthermore, less than $10 \%$ cases survived more than 2 years. However, from the review of previous reports concerning pneumothorax from epithelioid sarcomas, tumor progression with cystic pulmonary metastases of epithelioid sarcoma may be less aggressive. Only one case (7) resulted in death within 3 months due to massive pleural effusion from the first visit. Besides this case, most patients survive for several years even if a pneumothorax due to multiple pulmonary metastases is found. In the present case, the patient was alive 24 months after the development of a spontaneous pneumothorax.

At the follow-up, lung metastasis from sarcoma is common. Close lung follow-up is required by the orthopedic oncologist. Pulmonary metastasis from sarcomas is generally well-circumscribed and solid nodules. However, whether the lung nodule is cystic or non-cystic, careful attention must be paid every three months at least when the nodule size and numbers of are increasing in the lung.
In conclusion, pulmonary metastases forming small, solid, rounded peripheral nodules from soft tissue sarcoma are very common, but cystic pulmonary metastases from epithelioid sarcomas are extremely rare. Additionally, pneumothorax formation is a very common complication associated with cystic pulmonary metastases. For orthopedic oncologists, close observation is mandatory to gain awareness of this serious complication during follow-up in the advanced stage of epithelioid sarcoma. Tumor progression after pneumothorax appears to be less aggressive compared to that for pneumothorax of other sarcoma types.

\section{Acknowledgements}

We would like to thank Yuko Kuwae for pathological examinations and interpretations.

\section{References}

1. Hasegawa S, Inui K, Kamakari K, Kotoura Y, Suzuki K and Fukumoto M: Pulmonary cysts as the sole metastatic manifestation of soft tissue sarcoma: Case report and consideration of the pathogenesis. Chest 116: 263-265, 1999.

2. Chan DP, Griffith JF, Lee TW, Chow LT and Yim AP: Cystic pulmonary metastases from epithelioid cell sarcoma. Ann Thorac Surg 75: 1652-1654, 2003.

3. Kikuchi E, Kinoshita I, Yamazaki K, Itoh T, Shimizu T, Shimizu $\mathrm{H}$ and Nishimura M: Epithelioid sarcoma presenting as pulmonary cysts with cancer antigen 125 expression. Respirology 11: 826-829, 2006.

4. Choi SY, Kim YH, Kwon JB, Suh JH, Shin OR and Hong SH: Epithelioid sarcoma metastatic to the lung as pulmonary cysts without other metastatic manifestation. J Thorac Oncol 3: $532-533,2008$

5. Liu Y, Ma X, Zang D, Zhou C and Zhang J: Epithelioid sarcoma with osteoporosis and pneumothorax. Eur J Dermatol 21: 296-297, 2011.

6. Chong SG and Casserly BP: An unusual cause of spontaneous recurrent pneumothoraces. Br J Hosp Med (Lond) 73: 110-111, 2012.

7. Jeon SY, Yhim HY and Lee NR: Epithelioid sarcoma with spontaneous pneumothorax and massive pleural effusion. Korean J Intern Med 31: 191-193, 2016.

8. Ross HM, Lewis JJ, Woodruff JM and Brennan MF: Epithelioid sarcoma: Clinical behavior and prognostic factors of survival. Ann Surg Oncol 4: 491-495, 1997.

9. Livi L, Shah N, Paiar F, Fisher C, Judson I, Moskovic E, Thomas $\mathrm{M}$ and Harmer C: Treatment of epithelioid sarcoma at the royal marsden hospital. Sarcoma 7: 149-152, 2003.

10. Oda Y, Cin PD and Laskin WB: Epithelioid sarcoma. In: Fletcher CDM, Bridge JA, Hogendoorn PCW, Mertens F, (eds.). World Health Organization Classification of Tumors of Soft Tissue and Bone, Lyon, International Agency for Research on Cancer: 216-217, 2013.

11. Corrin B, Liebow AA and Friedman PJ: Pulmonary lymphangiomyomatosis. A review. Am J Pathol 79: 348-382, 1975.

12. Mark EJ: Mesenchymal cystic hamartoma of the lung. N Engl J Med 315: 1255-1259, 1986.

13. Hoag JB, Sherman M, Fasihuddin Q and Lund ME: A comprehensive review of spontaneous pneumothorax complicating sarcoma. Chest 138: 510-518, 2010.

14. Traweek T, Rotter AJ, Swartz W and Azumi N: Cystic pulmonary metastatic sarcoma. Cancer 65: 1805-1811, 1990.

15. Somasekharan Nair KK, Zabell AS, Vo KL and Shaikh MA: Pneumothorax: A classical presentation of metastatic scalp angiosarcoma. Ann Thorac Surg 94: e77-e78, 2012. 